4-1-2015

\title{
Inpatient management of migraine
}

\author{
Michael J. Marmura \\ Thomas Jefferson University \\ Stephanie Wrobel Goldberg \\ Thomas Jefferson University
}

Follow this and additional works at: https://jdc.jefferson.edu/neurologyfp

Part of the Medicine and Health Sciences Commons

Let us know how access to this document benefits you

\section{Recommended Citation}

Marmura, Michael J. and Goldberg, Stephanie Wrobel, "Inpatient management of migraine" (2015). Department of Neurology Faculty Papers. Paper 101.

https://jdc.jefferson.edu/neurologyfp/101

This Article is brought to you for free and open access by the Jefferson Digital Commons. The Jefferson Digital Commons is a service of Thomas Jefferson University's Center for Teaching and Learning (CTL). The Commons is a showcase for Jefferson books and journals, peer-reviewed scholarly publications, unique historical collections from the University archives, and teaching tools. The Jefferson Digital Commons allows researchers and interested readers anywhere in the world to learn about and keep up to date with Jefferson scholarship. This article has been accepted for inclusion in Department of Neurology Faculty Papers by an authorized administrator of the Jefferson Digital Commons. For more information, please contact: JeffersonDigitalCommons@jefferson.edu. 
Inpatient Management of Migraine:

Michael J Marmura, MD, FAHS

Stephanie Wrobel Goldberg, MD

Corresponding author:

Michael J Marmura

Thomas Jefferson Univerisity, Department of Neurology, Jefferson Headache Center

900 Walnut St Suite 200

Philadelphia PA 19107

$2159552243(\mathrm{P})$

$2159552060(\mathrm{~F})$

Michael.marmura@jefferson.edu

Keywords:

Migraine, inpatient, cognitive-behavioral therapy, chronic migraine, refractory migraine, dihydroergotamine.

Disclosure:

Dr. Marmura has received royalties from Medlink Neurology, Demos Medical and Cambridge University Press for publications. Dr. Goldberg has nothing to disclose. 


\begin{abstract}
:
Migraine is a frequently disabling disorder which may require inpatient treatment. Admission criteria for migraine include intractable migraine, nausea and/or vomiting, severe disability and dependence on opioids or barbiturates. The inpatient treatment of migraine is based on observational studies and expert opinion rather than placebo-controlled trials. Well-established inpatient treatments for migraine include dihydroergotamine, neuroleptics/antiemetics, lidocaine, intravenous aspirin and non-pharmacologic treatment such as cognitive-behavioral therapy. Short-acting treatments possibly associated with medication-overuse, such as triptans, opioids or barbiturate-containing compounds, are generally avoided. While the majority of persons with migraine are admitted on an emergency basis for only a few days, outcome studies and infusion protocols during elective admissions at tertiary headache centers suggest a longer length-of-stay may be needed for persons with intractable migraine.
\end{abstract}

\title{
Keywords:
}

Migraine, inpatient, chronic migraine, migraine pharmacology, hospitalization, chronic daily headache.

\section{Introduction:}

Migraine is a highly prevalent headache disease which typically affects patients during their most productive years. In the Global Burden of Disease Survey 2010, it was ranked as the third most prevalent disorder and seventh-highest specific cause of disability worldwide [1]. In the US population over 11 million persons suffer from migraine headache with moderate to severe disability [2]. From 1980 through 1989, the prevalence of chronic migraine headaches in the United States increased nearly 60\%, from 25.8 per 1000 persons to 41.0 per 1000 persons [3]. 
Although the vast majority of patients with migraine receive outpatient treatment alone, inpatient migraine treatment accounts for a substantial percentage of the overall cost of the disorder. In 2008 over 50 thousand patients were hospitalized for migraine in the US, representing $63 \%$ of all admissions for headache with a cost of $\$ 375$ million, roughly $\$ 7500$ per patient [4]. Lucado et al. reviewed emergency department (ED) visits and inpatient admissions for headache in the US using data from the Healthcare Cost and Utilization Project. Over 3 million persons visited the ED for headache in 2008, with migraine the most common diagnosis. Migraine was also the most common diagnosis leading to admission. The average length of stay of patients with a primary diagnosis of headache was 2.7 days, less than the average for all admissions, 4.6 days [5].

Inadequately treated migraine can lead to frequent ED visits, unneeded diagnostic testing, and severe economic burden due to loss of functioning [6]. Missing or impaired function at work accounts for most of the economic impact of migraine [7]. A small minority of persons with migraine account for the majority of missed work days [8]. A retrospective review showed that migraineurs generated nearly twice as many medical claims as comparison group patients with higher use of emergency services. Total medical and pharmacy claims costs were \$3.4 million for the migraine group compared to $\$ 2.1$ million for the comparison group. Diagnostic procedures in persons with migraine generated a yearly cost of $\$ 83,537$ compared to $\$ 13,140$ in the comparison group [9]. Using a cost-responsiveness analysis, chronic headache sufferers cost $187 \%$ more than patients who do not suffer from headache, surpassing other major chronic conditions such as diabetes and heart disease with a 176\% and 136\% cost increment respectively [10]. 
Overuse of acute medications frequently complicates migraine treatment. Opioids and barbiturates are particularly linked to migraine progression [11]. Headache sufferers are more likely to worsen from overusing abortive medications when compared to other patients with chronic pain [12]. Discontinuation of these medications is imperative for improvement but may produce profound acute withdrawal, including the risk of seizure from barbiturate discontinuation, making outpatient treatment very difficult [13]. Besides the obvious goals of reducing suffering and improving function, effective inpatient treatment for migraine can eliminate medication overuse [14]. Patients overusing acute medications such as opioids, caffeine or barbiturate-containing compounders have greater psychiatric comorbidity such as major depressive disorder, panic or anxiety disorders [15]. Inpatient treatment can be necessary for patients dependent on these medications with progressive or intractable migraine.

\section{Initial Evaluation and Admission Criteria:}

Most patients with migraine are successfully treated as an outpatient by using appropriate acute and preventive medications, lifestyle changes, psychological therapy and interventional procedures such as behavioral therapies, dietary restrictions, health enhancement activities, interventional treatments such as neurotoxin treatments, trigger point injections, nerve blocks and devices [16].

An increasing number of outpatient infusion centers and comprehensive outpatient treatment programs have emerged, providing an even broader dimension of outpatient level care. [17-19] . As with most disorders it is essential to matching the intensity of service to severity of illness. Diener and colleagues summarized the experience of several integrated care settings in Europe 
and USA and concluded that integrated multidisciplinary headache care improves outcomes and decrease costs with fewer unnecessary diagnostic testing and emergency department visits [20].

Many of these patients require a more intense and controlled environment of care during treatment than cannot be safely provided in an outpatient setting. Factors that determine the optimal setting, methods, and results of such treatment have come under increasing scrutiny secondary to the cost of in-patient care [21] Several expert surveys have attempted to define the conditions requiring inpatient hospitalization. A recent survey distributed among American Headache Society members showed that nausea and vomiting were the most popular factor favoring inpatient admission (63.4\%) followed by medication overuse headache with opioids $(61.3 \%)$ or barbiturates $(58.1 \%)$. Interestingly, the majority of participants did not consider overuse of triptans and NSAIDs alone to warrant admission [22]. Freitag et al [21] reviewed and summarized admission criteria for migraine and other headache. Based on multiple expert criteria [23,24] the most important indications requiring inpatient hospitalization for migraine are as follows:

- Status migranosus

- Dehydration or intractable nausea and/or vomiting

- Dependence on opioids, barbituates requiring detoxification

- Treatment or medical disease requiring careful observation

- Failed outpatient treatment or frequent ED visits requiring inpatient pain or psychiatric management

Villareal performed a comparative study between 212 out patients and 222 inpatients and analyzed variables such as anxiety, depression, self-rating pain, analgesic medication use and 
length of headache history as potential risk factors for hospitalization. The conclusion was that inpatients scored significantly higher on anxiety and depression scale, rated higher pain scales and used more analgesics than the outpatient counterparts. Identifying risk factors to guide health care providers to assign patients to an inpatient status in advance can reduce futile treatment and its costs [25].

The inpatient treatment of migraine typically involves admission through an emergency department. For patients with long-standing intractable migraine, however, a longer length of stay may be required to successfully treat the disorder. Studies reviewing hospitalization at specialized headache centers report longer lengths of stay with a median of up 13 days. Treatment of long-standing migraine in the inpatient setting improves pain, alleviates disability and reduces analgesic use, with long-term benefit [26,27]. In fact patients with medicationoveruse, specifically triptans, often improve the most [26]. Although most patients will continue to experience headache or migraine a small number, approximately $10 \%$, may remain headache free $[27,28]$ The majority of specialized centers utilize both medications and psychological services to treat migraine in the inpatient setting.

\section{Inpatient migraine treatment in adults:}

Multiple options for inpatient treatment of migraine exist, though the literature is largely uncontrolled and observational. Among the most utilized are intravenous medications including dihydroergotamine, neuroleptics, diphenhydramine, non-steroidal anti-inflammatory drugs (NSAIDs), anticonvulsants and magnesium. In addition to facilitating infusion treatments of migraine, inpatient care allows for withdrawal of overused medications such as opioids or barbiturates with close monitoring and treatment of withdrawal symptoms [26]. Comprehensive 
headache care centers use a multi-disciplinary approach combining pharmacologic treatment with behavioral therapies such as biofeedback, psychotherapy, or relaxation techniques, sometimes continued after discharge $[6,27,28]$.

\section{Pharmacologic treatment:}

1. Dihydroergotamine (DHE). Repetitive dosing of DHE to treat refractory migraine was first described by Raskin [29]. Fifty-five patients with continuous headache for at least two months, most with medication overuse, were treated with between $0.3 \mathrm{mg}$ and $1 \mathrm{mg}$ of intravenous DHE every 8 hours for 2 days. Forty-nine patients (89\%) were headache-free within 48 hours, with most reporting lasting benefit at follow-up.

Long term prognosis following in patient treatment with IV DHE has also been evaluated. Silberstein and colleagues retrospectively studied the 2 year outcome of 50 patients suffering from chronic daily headache $(\mathrm{CDH})$ associated with the overuse of analgesics admitted to their inpatient headache center. All 50 patients had some sort of chronic daily headache including chronic or transformed migraine (37), new daily persistent headache (NDPH) (12) and chronic tension-type headache (1). All patients suffered from overuse of either or combination of analgesics (narcotic and non-narcotic), caffeine, butalbital and ergotamine. At 3 months follow up, $44 \%$ of patients had an excellent or good result (excellent defined by $>90 \%$ reduction in either headache intensity or frequency or $>75 \%$ reduction in both; good defined by $>75 \%$ reduction in either headache intensity or frequency or $>50 \%$ reduction in both). At 6 months follow up 56\% had a good or excellent result. At 24 months ( 2 years follow up) 59\% of patients 
remained showing an excellent or good result. The authors concluded that most patients (87\%) continued to show significant improvement after 2 years of hospital admission [30].

Other chart review studies confirm long-term benefit after inpatient DHE treatment [31].

Nagy reported 114 patients with chronic migraine treated with a total dose of between $8.25 \mathrm{mg}$ and $11.25 \mathrm{mg}$ of intravenous DHE, $84(74 \%)$ reported at least some subjective benefit, 76 (67\%) reported headache freedom during treatment, and 85 (75\%) reported headache freedom within one month following treatment [32]. Both headache frequency and severity were reduced and the effect lasted an average of 28 days. Higher total DHE dose and less nausea were significant predictors of headache freedom.

Jauslin and Goadsby [33] evaluated 47 patients with migraine hospitalized for the management of severe headache. Of the 47 patients, 17 had status migrainosus and 12 had chronic daily headaches qualitatively of a migraine type. Treatment in all comprised cessation of all previous acute medication, plus one of the following: intravenous dihydroergotamine (DHE) 0.5mg eight hourly (7 patients), intravenous injection of lidocaine (lignocaine) with a bolus of $1 \mathrm{mg} / \mathrm{Kg}$ being followed by an infusion of $2 \mathrm{mg} / \mathrm{minute}$ for two days (11 patients), combination of these two techniques (18 patients) and subcutaneous injection of sumatriptan $3 \mathrm{mg}$, repeated in 30 minutes if necessary (8 patients). Of 12 patients treated with i.v. DHE, lidocaine infusion or both, 8 were headache-free within 2 days, 2 improved and 2 were unchanged. In contrast 5 of the 6 patients treated with sumatriptan were headache-free within 2 hours.

Relja and colleagues evaluated 101 patients who met ICHD 2 criteria for MOH and attempted to identify predictors of treatment efficacy. The time elapsed from the beginning of the detoxification to the resolution of headache (referred as TCDHR) was also evaluated as a way of 
measuring efficacy of the therapeutic protocol. The results confirmed that migraine without aura was the most common primary headache associated with medication overuse (75 of 101 patients). The use of IV DHE co administered with metoclopramide proved to be safe and effective and at 3 months follow up no patient relapsed to overusing medication. Patients overusing triptans had a faster resolution of their headaches. The mean length of hospital stay in this study was 10 days [34].

2. Lysine acetylsalicylate (aspirin). A few studies have shown intravenous aspirin to be an effective treatment for acute migraine attacks $[35,36]$ but only one has examined its use in an inpatient setting, given primarily for medication withdrawal headache [37]. In this retrospective review of 91 patients with migraine, one gram of intravenous aspirin had a moderate effect in $62 \%$ and good effect in $27 \%$. Intravenous aspirin was well-tolerated, with only minor adverse events reported. Of note, of 21 patients with a history of upper gastrointestinal problems or NSAID intolerance, only $3(14 \%)$ reported nausea or abdominal pain with intravenous aspirin.

3. Valproate. One uncontrolled study showed that eight $(80 \%)$ of ten patients with chronic migraine treated with a loading dose of $15 \mathrm{mg} / \mathrm{Kg}$ followed by $5 \mathrm{mg} / \mathrm{Kg}$ every 8 hours of intravenous valproate every eight hours had improvement of the pain. 57\% was pain free [38]. Intravenous valproate may also be effective for acute migraine in the emergency department and outpatient settings [39-41].

4. Lidocaine. Two retrospective reviews have examined the use of intravenous lidocaine for chronic migraine. Of 71 patients with chronic daily headache (90\% with migraine) and medication overuse treated with lidocaine infusion at $2 \mathrm{mg} / \mathrm{min}$ for seven to ten days, $90 \%$ noted improvement in headache by discharge, with $60 \%$ achieving headache freedom [42]. Six months 
later, headache was absent in 51\% and improved in an additional 20\%. Of 68 patients with chronic daily headache ( $60 \%$ with migraine) treated with between $1 \mathrm{mg} / \mathrm{min}$ to $4 \mathrm{mg} / \mathrm{min}$ of lidocaine for a mean of 8.5 days, $57 \%$ had some improvement and 25\% achieved headache freedom [43]. Most patients had received intravenous DHE, neuroleptics or corticosteroids during the hospitalization prior to lidocaine.

Side effects were generally mild, including nausea, hypotension, and arrhythmia, and did not lead to treatment discontinuation. However, hallucinations [44] and other psychiatric side effects are not uncommon [45].

5. Magnesium. The data available regarding the use of intravenous magnesium for the treatment of headache in adults is conflicting, most reports focusing on its use in the emergency setting rather than in patient and for the treatment of migraine headaches. Bigal et al. assessed the effect of $1 \mathrm{~g}$ intravenous magnesium sulphate on the pain and associated symptoms (nausea, photophobia and phonophobia) in patients with migraine without aura and migraine with aura compared to placebo. In the migraine without aura group there was no statistically significant difference in the patients who received magnesium sulphate vs. placebo in pain relief. In the migraine with aura group though patients receiving magnesium sulphate presented a statistically significant improvement of pain and of all associated symptoms compared with controls. Data supports use of magnesium sulphate for the treatment of all symptoms in migraine with aura, or as an adjuvant therapy for associated symptoms in patients with migraine without aura [46].

In a randomized, single-blind, placebo-controlled trial 15 patients received $1 \mathrm{~g}$ intravenous magnesium sulfate given and were compared to 15 patients who received $10 \mathrm{~mL}$ of $0.9 \%$ saline 
intravenously. Magnesium sulfate was found to be superior to placebo with a pain-free rate of $87 \%$ for magnesium sulfate compared to $0 \%$ for placebo [47].

Frank et al. published a randomized double-blind placebo-controlled trial comparing $2 \mathrm{~g}$ of IV magnesium versus placebo for the treatment of 42 patients ( 21 in each treatment group) with acute benign headache who presented to the EDs of two teaching hospitals. Authors found no benefit of IV magnesium compared to placebo [48].

A meta-analysis of trials including 295 patients treated with intravenous magnesium for acute migraine failed to demonstrate a beneficial effect in terms of reduction in pain and decrease for rescue medication. It also showed that patients treated with magnesium were significantly more likely to report adverse events [49].

In one study metoclopramide was more effective than magnesium with statistically significant differences [50]. In another study comparing a neuroleptic agent (prochlorperazine) and magnesium, the results were not statistically significant [51]. A small case series reviewed the effect of intravenous magnesium therapy for 20 adolescent patients (range 13-18 years old): 5 with migraine, 4 tension-type headache, and 11 with status migrainosus. Although the treatment showed good tolerability there was no clear efficacy, although the study was limited by small sample size, multiple potential confounders, and lack of controls. There are no available controlled studies in the pediatric population.

6. Diphenhydramine. The role of histamine in the pathophysiology of migraine is still under investigation but studies suggest benefits from intravenous administration. A study by Somerville [52] reported that the administration of an acetaminophen-codeine combination plus the antihistamine doxylamine significantly relieved acute migraine attacks compared with 
placebo. Swidan and colleagues compared the efficacy of intravenous diphenhydramine with dihydroergotamine mesylate (DHE) in the treatment of severe, refractory, migraine headache in eighty patients divided into two groups of 40 patients each. Comparison was made between nine doses of diphenhydramine ( 25 to $75 \mathrm{mg}$ administered intravenously three times daily) and nine doses of DHE (0.25 to $1.0 \mathrm{mg}$ administered intravenously approximately every 8 hours) during a 3-day period. Patients receiving DHE also received 10 to $15 \mathrm{mg}$ of metoclopramide administered orally or intravenously approximately 30 minutes before the DHE for nausea prophylaxis. Intravenous diphenhydramine was noted to reduce pain levels more effectively than DHE immediately after its administration although overall DHE was associated with greater reduction in head pain level after the full nine doses (pre- and post protocol pain scores were compared). Despite the study's limitations authors concluded that diphenhydramine could be effective in the treatment of some patients with intractable chronic migraine [53].

7. Antiemetics. The majority of available data available mostly focus on outcome of treatment in the emergency department or infusion settings. According to a systematic review from the Canadian Headache Society, intravenous prochlorperazine is strongly recommended at a dose of $10 \mathrm{mg}$ as high quality evidence for acute ttreatment of migraine [54]. Multiple clinical trials have showed superior effect compared to placebo, SC sumatriptan and sodium valproate [54-57]. Intravenous droperidol [58,59] is effective in status migranosus and more effective than placebo in controlled trials for acute migraine. Multiple studies also support the use of chlorpromazine [60,61], haloperidol [62], and metoclopramide $[63,64]$ with or in conjunction with DHE treatment. EKG monitoring is recommended for drugs which may prolong QT interval. 
8. Propofol. Intravenous propofol in both subanesthetic and sedating doses has been reported to be effective for acute migraine in outpatient [65] and emergency department settings $[66,67]$. In a small study conducted in an inpatient setting for airway monitoring, 18 patients with chronic daily headache (14 with chronic migraine) were treated with repetitive boluses of propofol with a mean total dose of 234mg [68]. Six achieved headache freedom and 11 reported reduced headache intensity with a mean decrease of 4.2 points on a 10 point scale. There were no adverse events other than drowsiness, and in fact patients who slept between boluses had more pain relief.

\section{Inpatient migraine treatment in children:}

Children account for about 1/3 of ED visits and the prevalence of headache as a chief complaint in pediatric EDs is similar to adults [69]. In children presenting with headache as a chief complaint in the ED, migraine is the second most common etiology following viral upper respiratory tract infection [70]. Although a careful history is essential, serious secondary headaches in children usually present with clear neurological abnormalities on exam [71]. A substantial minority of children with migraine fails to improve with emergency treatment and may be candidates for inpatient management [72]. Given that migraine causes severe disability and that children are expected to attend school regularly, untreated migraine in children may have profound implications. The treatment of children with migraine and other headache is largely drawn from protocols used in adults. Many treatments used for adults with headache have not been adequately studied in children, such as the use of corticosteroids. There have been a few significant pediatric studies of inpatient headache treatment, and other adult studies which have included adolescents. 
A recent survey of headache specialists asked respondents to list their preferred treatments based on a clinical case of a 14 year-old girl with uncomplicated migraine [22]. Preferred treatments included, in order, NSAIDs, dopamine antagonists, hydration, IV magnesium, and_triptans. Respondents selected DHE, corticosteroids and valproic acid more often as second line treatments. Opioids and barbiturate-containing medications were not recommended.

Linder reported the successful inpatient use of combined metoclopramide and dihydroergotamine for children aged 6 and older. Patients who had previously failed outpatient treatment received oral metoclopramide followed by intravenous DHE. Linder selected oral metoclopramide after observing side effects such as sedation, akathisia and extrapyramidal effects with intravenous prochlorperazine, metoclopramide, and promethazine. The protocol for inpatient included metoclopramide $0.2 \mathrm{mg} / \mathrm{kg} 30$ minutes prior to DHE doses adjusted for age (0.1 mg age 6-9, 0.15 mg age 9-12, $0.2 \mathrm{mg}$ age 12-16) every 6 hours, with the ability to increase dose by $0.05 \mathrm{mg}$ each time if tolerated up to a maximum of $0.5 \mathrm{mg} / \mathrm{dose}$. Of 30 children, 24 reported an excellent response (90\% improvement of either headache frequency or intensity or $75 \%$ improvement of both) [28].

Kabbouche reported the use of prochlorperazine $0.13-15 \mathrm{mg} / \mathrm{kg}$ with migraine using a higher dose of DHE 0.5-1 mg every 8 hours in pediatric inpatients with migraine [72,73]. To prevent the development of extrapyramidal effects, prochlorperazine was changed to another neuroleptic after 3 doses. After 10 doses, 97\% of children reported improvement and $77 \%$ headache freedom.

Nagy et al. [32] reported the inpatient use of DHE in patients with multiple headache disorders such as migraine, cluster headache and new daily persistent headache over 5 days including 
children. In their pediatric patients under 16 they dosed DHE by weight in kilograms, using intravenous ondansetron $4 \mathrm{mg} 30$ minutes prior to DHE and giving an extra $4 \mathrm{mg}$ or an alternative antinausea medication such as promethazine for those with significant nausea. Although they did not report pediatric outcomes separately, patients with migraine, cluster headache and NPDH with migraine features often improved both during the treatment with continued improvement after discharge.

Raina [74] reported a case series describing the use of DHE for the treatment of 6 children, 5 of them female, aged 13-19 with likely abdominal migraine. Patients initially received $0.5 \mathrm{mg}$ and a total of 7-11 mg during their hospitalizations. Nausea was a very common side effect.

Lake et al [75] reported a prospective outcome study in inpatients with headache with an age range of 13-73. After an structured inpatient program consisting of intravenous dihydroergotamine mesylate or hydrocortisone over 3 days, combined with clinical psychological evaluation and follow-up, over $60 \%$ of patients reported at least $50 \%$ improvement in severe headache days with lower rates of acute medication use, and improved sleep and functional performance. Pediatric outcomes were included in the overall outcomes.

Two case series including adolescents have $[42,43]$ reported the inpatient use of intravenous lidocaine for the treatment of chronic daily headache with most of those having chronic migraine. Williams and Stark reviewed outcomes in those aged 16-74 over a period of 3-17 days while Rosen et al. studied those aged 17-66 years old over a period of 2-15 days. Patients received a dose of $2 \mathrm{mg} / \mathrm{minute}$ if tolerated. Further increases were based on tolerability, serum lidocaine levels and headache improvement. Although the majority of patients in both series 
reported complete or partial improvement, even those with medication overuse, pediatric outcomes were not reported separately.

Lanzi and colleagues [76] reported an open-label comparison of inpatient admission compared with outpatient care for children with at least 2 months of moderate-severe migraine or tensiontype headache. During hospitalizations, children and their families received psychological care including 3 individual interviews focusing on fears, family and social relationships and behavioral problems. In addition a child and adolescent psychiatrist assessed hospitalized child for co-morbid conditions. Neither group of children received prophylactic treatment for migraine. At 1, 3 and 6 months, those children who were hospitalized had significantly fewer attacks, less severe attacks and less medication use compared to those who had outpatient care.

Heschler et al. [77] reported outcomes from a 3-week multimodal inpatient pain program for children and adolescents with chronic pain, with headache being the most common complaint among these subjects $(84 / 167,50 \%)$ followed by recurrent abdominal pain $(22 / 167,13 \%)$. At baseline, $42 \%$ of patients reported severe pain and $55 \%$ very severe pain. A multiprofessional team including pediatricians, clinical psychologists, children and adolescent psychiatrists, pediatric nurses, physiotherapists, occupational therapists, and social workers evaluated these children and initiated treatment which included psychotherapy, cognitive-behavioral therapy, development of pain-coping strategies, family therapy, music and art therapy. After 3 month follow-up, treated children made significant improvements in pain intensity, school absence and acute medication use.

\section{Conclusion:}


The effective inpatient treatment of migraine involves both acute medication to reduce pain and preventive treatments to improve long-term outcomes. The general principles of inpatient management are listed in Table 1. The majority of persons admitted for migraine are admitted on an emergency basis for short periods of time to address relatively acute problems. However, based on the above studies involving large tertiary headache centers, most of the protocols for intractable migraine require a much longer length of stay. Prior to admission it is important to address the goals and priorities of admission. This should include issues such as school or work disability, family and plans for after discharge. For patients with medication-overuse, especially those using opioids or barbiturates, decreasing their daily dose prior to admission will allow a more successful treatment, as detoxification from very high doses may not be possible during a relatively brief hospitalization. An inpatient hospitalization offers the opportunity to strengthen therapeutic relationships with patients, observe family dynamics and relationships, and provide education about migraine and chronic pain. Diagnostic testing, when indicated, can rule out important secondary causes of headache. Given that many patients with secondary headache such as idiopathic intracranial hypertension [78] have features of migraine, lumbar puncture may be necessary.

The medications used to treat migraine in the inpatient setting are somewhat different than those recommended in the outpatient setting as established by clinical trials [79]. Most migraine clinical trials focus on acute migraine and exclude those with chronic migraine and medicationoveruse. Short-acting medications such as triptans and opioids should not typically be used for inpatient migraine, especially in those with intractable disease or medication-overuse as an outpatient. Inpatient treatment of migraine often involves medications which require special monitoring: DHE may increase blood pressure and monitoring blood pressure is recommended; 
many neuroleptics are associated with prolonged QT interval requiring frequent electrocardiograms; prolonged use of intravenous lidocaine may require telemetry monitoring or drug levels. A knowledgeable nursing staff familiar with migraine, common adverse events and the administration of these medications is very helpful.

The use of ancillary services is important for the management of intractable headache disorders. Multiple affective disorders are strongly linked with chronic migraine, including depression, anxiety and bipolar spectrum disorders [80]. Abuse and maltreatment, especially early in life, are strongly linked with migraine chronification [81]. Given that several studies have described the effectiveness of mental health treatment in improving long-term outcomes in pain disorders, a psychologist with interest in pain disorders should be part of the migraine treatment team. Physical therapy may also be useful as neck pain and muscle spasm are extremely common in persons with migraine. Given the strong association with obesity and migraine chronification [82], nutrition consultant may improve long-term outcomes, especially given recent evidence that long-term weight loss appears to improve migraine severity [83].

Patients receiving inpatient treatment for migraine will continue to require close follow-up after discharge. Almost all will need at least one preventive medication treatment, and may need to continue titrating medications started during or before the hospitalization. Because most preventives work best over time, it is important to ensure that patients continue their preventive medications after discharge. At the time of discharge, a written plan should explain how to treat migraine with acute medications when they do occur using the principles of stratified care [84].

Often response to treatment in the hospital will guide acute care after discharge. In most patients, this will mean not taking medication for mild headaches and using an NSAID, antiemetics, DHE 
or triptans at migraine onset. Neuroleptics and other sedating medications may be considered as a rescue treatment. Opioids and corticosteroids should generally be avoided for regular use but may be considered for special situations in which other medications are contraindicated. A nonoral option may worthwhile, especially in patients who either overuse oral medications or with frequent nausea or vomiting. Discharging providers should provide referrals to appropriate providers such as psychologists, psychiatrists, physical therapy or nutrition at the time of discharge. If possible having a follow-up appointment before discharge scheduled no more than a few weeks later is ideal.

Table 1: Principles for inpatient migraine treatment

\begin{tabular}{|l|l|}
\hline Carefully titrate medications to effective doses & Withdraw opioids and barbiturates if possible \\
daily headache such as DHE & Monitor EKG, blood pressure, and consider \\
\hline Address medication-overuse if present & Titrate preventive medication \\
\hline $\begin{array}{l}\text { Utilize mental health providers to assist with } \\
\text { pain-coping strategies }\end{array}$ & Provide a clear discharge plan for acute \\
\end{tabular}

References

Papers of particular interest, published recently, have been highlighted as:

- Of importance

•• Of major importance

1. MURRAY CJ, LOPEZ AD, BLACK R, et al: Global burden of disease 2005: call for collaborators. Lancet (2007) 370(9582):109-110. 
2. STEWART WF, LIPTON RB, CELENTANO DD, REED ML: Prevalence of migraine headache in the United States. Relation to age, income, race and other sociodemographic factors. JAMA (1992) 26764-69.

3. AnonymousFrom the Centers for Disease Control. Prevalence of chronic migraine headaches-United States, 1980-1989. JAMA (1991) 265(22):2941

4. INSINGA RP, NG-MAK DS, HANSON ME: Costs associated with outpatient, emergency room and inpatient care for migraine in the USA. Cephalalgia (2011) 31(15):1570-1575. A

5. - LUCADO J, PAEZ K, ELIXHAUSER A: Headaches in U.S. Hospitals and Emergency Departments, 2008: Statistical Brief \#111. (2006) A relatively unusual look at actual costs of primary headache disorders as they are currently treated, using real-world data.

6. LAKE AE, SAPER JR, MADDEN SF, KREEGER C: Comprehensive inpatient treatment for intractable migraine: a prospective long-term outcome study. Headache (1993) (33):55-62.

7. HU XH, MARKSON LE, LIPTON RB, STEWART WF, BERGER ML: Burden of migraine in the United States: disability and economic costs. Arch Intern Med (1999) 159813-818.

8. VONKORFF M, STEWART WF, SIMON DS, LIPTON RB: Migraine and reduced work performance: a population-based diary study. Neurology (1998) 501741-1745.

9. OSTERHAUS J, GUTTERMAN DL, PLUCHETKA JR: Healthcare resource and lost labor costs of migraine headache in the United States. Pharmaco Economics (1992) 267-76.

10. FISHMAN P, VON KM, LOZANO P, HECHT J: Chronic care costs in managed care. Health Aff. (Millwood. ) (1997) 16(3):239-247.

11. BIGAL ME, LIPTON RB: Excessive opioid use and the development of chronic migraine. Pain (2009) 142(3):179-182.

12. FERRARI A, LEONE S, TACCHI R, et al: The link between pain patient and analgesic medication is greater in migraine than in rheumatic disease patients. Cephalalgia (2009) 29(1):31-37.

13. $\bullet$ SAPER JR, LAKE AE, III: Continuous opioid therapy (COT) is rarely advisable for refractory chronic daily headache: limited efficacy, risks, and proposed guidelines. Headache (2008) 48(6):838-849. A guideline for management of daily headache, pointing out common pitfalls with opioid use seen in clinical practice.

14. TRIBL GG, SCHNIDER P, WOBER C, et al: Are there predictive factors for long-term outcome after withdrawal in drug-induced chronic daily headache? Cephalalgia (2001) 21(6):691-696.

15. RADAT F, SAKH D, LUTZ G, et al: Psychiatric comorbidity is related to headache induced by chronic substance use in migraineurs. Headache (1999) 39(7):477-480.

16. SCHOENEN J, VANDERSMISSEN B, JEANGETTE S, et al: Migraine prevention with a supraorbital transcutaneous stimulator: a randomized controlled trial. Neurology (2013) 80(8):697-704. 
17. ZEEBERG P, OLESEN J, JENSEN R: Efficacy of multidisciplinary treatment in a tertiary referral headache centre. Cephalalgia (2005) 25(12):1159-1167.

18. - GAUL C, VISSCHER CM, BHOLA R, et al: Team players against headache: multidisciplinary treatment of primary headaches and medication overuse headache. $J$ Headache Pain (2011) 12(5):511-519. An excellent overview of comprehensive headache management.

19. RIZZOLI P: Preventive pharmacotherapy in migraine. Headache (2014) 54(2):364-369.

20. DIENER HC, GAUL C, JENSEN R, et al: Integrated headache care. Cephalalgia (2011) 31(9):10391047.

21. $\bullet$ FREITAG FG, LAKE A, LIPTON RB, et al: Inpatient treatment of headache: an evidence-based assessment. United States Headache Guidelines Consortium. Headache (2004) 44 342-360. The most complete evidence and experience-based review of inpatient headache management to date.

22. $\bullet$ DOUGHERTY CO, MARMURA MJ, ERGONUL Z, CHARLESTON LC, SZPERKA CL: Emergency and inpatient treatment of migraine: an AmericanHeadache Society survey. Brit J Medicine Medical Research (2014) 4(20):3800-3813. A recent expert opinion paper on the topic of emergency and inpatient management.

23. Saper JR, Silberstein SD, Gordon CD, et al. Handbook of headache management. In: Saper, J. R., Silberstein, S. D., Gordon, C. D., and Hamel, R. L.Handbook of headache management. Baltimore: Williams \& Wilkins; 1993.

24. SOLOMON GD, CADY RK, KLAPPER JA, RYAN RE, JR.: Standards of care for treating headache in primary care practice. National Headache Foundation. Cleve. Clin J Med (1997) 64(7):373-383.

25. VILLARREAL SS: A comparative study of selected patient variables as risk factors in hospitalization for chronic headache. Headache (1995) 35 349-354.

26. $\bullet$ LAKE AE, III, SAPER JR, HAMEL RL: Comprehensive inpatient treatment of refractory chronic daily headache. Headache (2009) 49(4):555-562. A recent overview of chronic daily headache management with insight from the medical and psychological prespective.

27. DIAMOND S, FREITAG FG, MALISZEWSKI M: Inpatient treatment of headache: long-term results. Headache (1986) 26 189-197.

28. LINDER SL: Treatment of acute childhood migraine headaches. Cephalalgia (1991) 11(Suppl 11):120-121.

29. RASKIN NH: Repetitive intravenous dihydroergotamine as therapy for intractable migraine. Neurology (1986) 36995-997.

30. SILBERSTEIN SD, SILBERSTEIN JR: Chronic daily headache: prognosis following inpatient treatment with repetitive IV DHE. Headache (1992) 32439-445.

31. PRINGSHEIM T, HOWSE D: Inpatient treatment of chronic daily headache using dihydroergotamine: a long-term followup study. Can J Neurol Sci (1998) 25146-150. 
32. - NAGY AJ, GANDHI S, BHOLA R, GOADSBY PJ: Intravenous dihydroergotamine for inpatient management of refractory primary headaches. Neurology (2011) 77(20):1827-1832. One of a few recent large inpatient studies of DHE.

33. JAUSLIN P, GOADSBY PJ, LANCE JW: The hospital management of severe migrainous headache. Headache (1991) 31658-660.

34. RELJA G, GRANATO A, BRATINA A, ANTONELLO RM, ZORZON M: Outcome of medication overuse headache after abrupt in-patient withdrawal. Cephalalgia (2006) 26(5):589-595.

35. Diener HC: The efficacy and safety of acetylsalicylic acid lysinate compared to subcutaneous sumatriptan and parenteral placebo in the acute treatment of migraine. A double-blind, doubledummy, randomized multicenter, parallel group study. The ASASUMAMIG Study Group. Eur Neurol 1999;(In Press)

36. LIPTON RB, GOLDSTEIN J, BAGGISH JS, et al: Aspirin is efficacious for the treatment of acute migraine. Headache (2005) 45(4):283-292.

37. - WEATHERALL MW, TELZEROW AJ, CITTADINI E, KAUBE H, GOADSBY PJ: Intravenous aspirin (Iysine acetylsalicylate) in the inpatient management of headache. Neurology (2010) 75(12):10981103. A novel inpatient approach to daily headache in the hospital setting.

38. SCHWARTZ TH, KARPITSKIY VV, SOHN RS: Intravenous valproate sodium in the treatment of daily headache. Headache (2002) 42(6):519-522.

39. STILLMAN MJ, ZAJAC D, RYBICKI LA: Treatment of primary headache disorders with intravenous valproate: initial outpatient experience. Headache (2004) 44(1):65-69.

40. MATHEW NT, KAILASAM J, MEADORS L, GENTRY P, CHERNYSHEV O: Intravenous valproate sodium (Depaconr) aborts migraine rapidly: a preliminary report. Cephalalgia (1999) 19:373 (Abstract).

41. EDWARDS KR, NORTON J, BEHNKE M: Comparison of intravenous valproate versus intramuscular dihydroergotamine and metoclopramide for acute treatment of migraine headache. Headache (2001) 41(10):976-980.

42. WILLIAMS DR, STARK RJ: Intravenous lignocaine (lidocaine) infusion for the treatment of chronic daily headache with substantial medication overuse. Cephalalgia (2003) 23(10):963-971.

43. - ROSEN N, MARMURA M, ABBAS M, SILBERSTEIN S: Intravenous lidocaine in the treatment of refractory headache: a retrospective case series. Headache (2009) 49(2):286-291. A large review of lidocaine for chronic migraine and other headaches in the inpatient setting, with or without adjunctive medication.

44. MARMURA MJ, PASSERO FC, JR., YOUNG WB: Mexiletine for refractory chronic daily headache: a report of nine cases. Headache (2008) 48(10):1506-1510.

45. GIL-GOUVEIA R, GOADSBY PJ: Neuropsychiatric side effects of lidocaine: examples from the treatment of headache and a review. Cephalalgia (2006) 26:1399 (Abstract). 
46. BIGAL ME, BORDINI CA, TEPPER SJ, SPECIALI JG: Intravenous magnesium sulphate in the acute treatment of migraine without aura and migraine with aura. A randomized, double-blind, placebocontrolled study. Cephalalgia (2002) 22(5):345-353.

47. DEMIRKAYA S, VURAL O, DORA B, TOPCUOGLU MA: Efficacy of intravenous magnesium sulfate in the treatment of acute migraine attacks. Headache (2001) 41(2):171-177.

48. FRANK LR, OLSON CM, SHULER KB, GHARIB SF: Intravenous magnesium for acute benign headache in the emergency department: a randomized double-blind placebo-controlled trial. CJEM. (2004) 6(5):327-332.

49. $\mathrm{CHOI} \mathrm{H}$, PARMAR $\mathrm{N}$ : The use of intravenous magnesium sulphate for acute migraine: metaanalysis of randomized controlled trials. Eur J Emerg. Med (2014) 21(1):2-9.

50. CETE Y, DORA B, ERTAN C, OZDEMIR C, OKTAY C: A randomized prospective placebo-controlled study of intravenous magnesium sulphate vs. metoclopramide in the management of acute migraine attacks in the Emergency Department. Cephalalgia (2005) 25(3):199-204.

51. GINDER S, OATMAN B, POLLACK M: A prospective study of i.v. magnesium and i.v. prochlorperazine in the treatment of headaches. J Emerg Med (2000) 18(3):311-315.

52. SOMERVILLE BW: Treatment of migraine attacks with an analgesic combination (Mersyndol). Med J Aust (1976) 1(23):865-866.

53. SWIDAN SZ, LAKE AE, III, SAPER JR: Efficacy of intravenous diphenhydramine versus intravenous DHE-45 in the treatment of severe migraine headache. Curr Pain Headache Rep. (2005) 9(1):65-70.

54. ORR SL, AUBE M, BECKER WJ, et al: Canadian Headache Society systematic review and recommendations on the treatment of migraine pain in emergency settings. Cephalalgia (2014)

55. KOSTIC MA, GUTIERREZ FJ, RIEG TS, MOORE TS, GENDRON RT: A prospective, randomized trial of intravenous prochlorperazine versus subcutaneous sumatriptan in acute migraine therapy in the emergency department. Ann Emerg. Med (2010) 56(1):1-6.

56. JONES EB, GONZALES ER, BOGGS JG, GRILLO JA, ELSWICK RK, JR.: Safety and efficacy of rectal prochlorperazine for the treatment of migraine in the emergency department. Ann Emerg Med (1994) 24237-241.

57. JONES J, SKLAR D, DOUGHERTY J, WHITE W: Randomized double-blind trial of intravenous prochlorperazine for the treatment of acute headache. JAMA (1989) 261(8):1174-1176.

58. WANG SJ, SILBERSTEIN SD, YOUNG WB: Droperidol treatment of status migrainosus and refractory migraine. Headache (1997) 37377-382.

59. SILBERSTEIN SD, YOUNG WB, MENDIZABAL JE, ROTHROCK JF, ALAM AS: Acute migraine treatment with droperidol: a randomized, double-blind, placebo-controlled trial. Neurology (2003) 60(2):315321. 
60. BIGAL ME, BORDINI CA, SPECIALI JG: Intravenous chlorpromazine in the emergency department treatment of migraines: a randomized controlled trial. J Emerg. Med. (2002) 23(2):141-148.

61. LANE PL, MCLELLAN BA, BOGGOLEY CJ: Comparative efficacy of chlorpromazine and meperidine with dimenhydrinate in migraine headache. Ann Emerg Med (1989) 18360-365.

62. HONKANIEMI J, LIIMATAINEN S, RAINESALO S, SULAVUORI S: Haloperidol in the acute treatment of migraine: a randomized, double-blind, placebo-controlled study. Headache (2006) 46(5):781787.

63. KLAPPER JA, STANTON JS: Ketorolac versus DHE and metoclopramide in the treatment of migraine headaches. Headache (1991) 31523-524.

64. TFELT-HANSEN P, OLESEN J, AEBELHOLT-KRABBE A, MELGAARD B, VEILIS B: A double blind study of metoclopramide in the treatment of migraine attacks. J Neurol Neurosurg Psychiatry (1980) 43369-371.

65. KRUSZ JC, SCOTT V, BELANGER J: Intravenous propofol: unique effeciveness in treating intractable migraine. Headache (2000) 40(3):224-230.

66. MOSIER J, ROPER G, HAYS D, GUISTO J: Sedative dosing of propofol for treatment of migraine headache in the emergency department: a case series. West J Emerg. Med (2013) 14(6):646-649.

67. SOLEIMANPOUR H, TAHERAGHDAM A, GHAFOURI RR, et al: Improvement of refractory migraine headache by propofol: case series. Int J Emerg. Med (2012) 5(1):19

68. - MENDES PM, SILBERSTEIN SD, YOUNG WB, ROZEN TD, PAOLONE MF: Intravenous Propofol in the Treatment of Refractory Headache. Headache: The Journal of Head and Face Pain (2002) 42(7):638-641. One of the largest studies looking at propofol for the treatment of intractable pain.

69. NELSON DS, WALSH K, FLEISHER GR: Spectrum and frequency of pediatric illness presenting to a general community hospital emergency department. Pediatrics (1992) 90(1 Pt 1):5-10.

70. KANDT RS, LEVINE RM: Headache and acute illness in children. J Child Neurol (1987) 2(1):22-27.

71. LEWIS DW, QURESHI F: Acute headache in children and adolescents presenting to the emergency department. Headache (2000) 40(3):200-203.

72. - KABBOUCHE MA, POWERS SW, SEGERS A, et al: Inpatient treatment of status migraine with dihydroergotamine in children and adolescents. Headache (2009) 49(1):106-109. One of only a few pediatric studies focusing on the treatment of chronic migraine.

73. KABBOUCHE MA, VOCKELL AL, LECATES SL, POWERS SW, HERSHEY AD: Tolerability and effectiveness of prochlorperazine for intractable migraine in children. Pediatrics (2001) 107(4):E62

74. RAINA M, CHELIMSKY G, CHELIMSKY T: Intravenous dihydroergotamine therapy for pediatric abdominal migraines. Clin Pediatr. (Phila) (2013) 52(10):918-921. 
75. LAKE A, SAPER J, MADDEN S, KREEGER C: Inpatient treatment for chronic daily headache: a prospective long-term outcome. Headache (1990) 30:299-300 (Abstract).

76. LANZI G, D'ARRIGO S, TERMINE C, et al: The effectiveness of hospitalization in the treatment of paediatric idiopathic headache patients. Psychopathology (2007) 40(1):1-7.

77. HECHLER T, DOBE M, KOSFELDER J, et al: Effectiveness of a 3-week multimodal inpatient pain treatment for adolescents suffering from chronic pain: statistical and clinical significance. Clin $J$ Pain (2009) 25(2):156-166.

78. WALL M, KUPERSMITH MJ, KIEBURTZ KD, et al: The idiopathic intracranial hypertension treatment trial: clinical profile at baseline. JAMA Neurol (2014) 71(6):693-701.

79. SILBERSTEIN SD: Practice Parameter--Evidence-based guidelines for migraine headache (an evidence-based review): Report of the Quality Standards Subcommittee of the American Academy of Neurology for the United States Headache Consortium. Neurology (2000) 55754-762.

80. BUSE DC, MANACK AN, FANNING KM, et al: Chronic migraine prevalence, disability, and sociodemographic factors: results from the American Migraine Prevalence and Prevention Study. Headache (2012) 521456-1470.

81. TIETJEN GE, BRANDES JL, PETERLIN BL, et al: Childhood maltreatment and migraine (part II). Emotional abuse as a risk factor for headache chronification. Headache (2010) 50(1):32-41.

82. PETERLIN BL, ROSSO AL, RAPOPORT AM, SCHER Al: Obesity and migraine: the effect of age, gender and adipose tissue distribution. Headache (2010) 50(1):52-62.

83. BOND DS, VITHIANANTHAN S, NASH JM, THOMAS JG, WING RR: Improvement of migraine headaches in severely obese patients after bariatric surgery. Neurology (2011) 76(13):1135-1138.

84. LIPTON RB, STEWART WF, SAWYER J: Stratified care is a more effective migraine treatment strategy than stepped care: results of a randomized clinical trial. Neurology (2000) 54A14 